\title{
Uranium Isotopes Separation Technique
}

\author{
Iman Tarik Al-Alawy*, Raghad Saadoon Mohammed, \\ Mohammed Zorah Hassan, Waleed Jabar Mhanah \\ Al-Mustansiriyah University College of Science, Physics department, Baghdad - Iraq \\ *E-mail address: drimantarik@yahoo.com
}

Keywords: Uranium isotopes, Laser separation, Enriched uranium.

\begin{abstract}
This work describes the atomic laser isotope separation (LIS). The (LIS) plant means calculating the values of a large number of parameters in order to optimize some objective function. Here we use simple model to describe the elementary physical processes: evaporation, vapor expansion, interaction between photons and atoms, ion extraction etc...concentrated on theoretical and empirical bases. An optimization process for the separation of Uranium isotopes is described and discussed.
\end{abstract}

\section{INTRODUCTION}

Naturally occurring uranium has three isotopes ${ }^{234} \mathrm{U},{ }^{235} \mathrm{U}$, and ${ }^{238} \mathrm{U}$. The amount of concentration of each natural isotope is $0.0055 \%, 0.7200 \%, 99.2745 \%$ respectively [1,2]. Uranium enriched to higher levels, as a nuclear explosive material in nuclear weapons. There are a number of different methods to enrich uranium. Enriched uranium is a type of uranium in which the percent composition of uranium-235 has been increased through the process of isotope separation. Natural uranium $238 \mathrm{U}$ isotope, with $235 \mathrm{U}$ only constituting about $0.711 \%$ of its weight. $235 \mathrm{U}$ is the only nuclide existing in nature (in any appreciable amount) that is fissile with thermal neutrons [3]. Enriched uranium is a critical component for both civil nuclear power generation and military nuclear weapons. The International Atomic Energy Agency attempts to monitor and control enriched uranium supplies and processes in its efforts to ensure nuclear power generation safety and curb nuclear weapons proliferation. The $238 \mathrm{U}$ remaining after enrichment is known as depleted uranium (DU), and is considerably less radioactive than even natural uranium, though still very dense and extremely hazardous in granulated form - such granules are a natural by-product of the shearing action that makes it useful for armor-penetrating weapons and radiation shielding. At present, 95 percent of the world's stocks of depleted uranium remain in secure storage. Lowenriched uranium (LEU) has a lower than $20 \%$ concentration of $235 \mathrm{U}$. For use in commercial light water reactors (LWR), the most prevalent power reactors in the world, uranium is enriched to 3 to $5 \% 235$ U. Fresh LEU used in research reactors is usually enriched $12 \%$ to $19.75 \%$ U-235, the latter concentration being used to replace HEU fuels when converting to LEU [4]. Highly enriched uranium (HEU) has a $20 \%$ or higher concentration of $235 \mathrm{U}$. The fissile uranium in nuclear weapon primaries usually contains $85 \%$ or more of $235 \mathrm{U}$ known as weapons-grade, though theoretically for an implosion design, a minimum of $20 \%$ could be sufficient (called weapon(s)-usable) although it would require hundreds of kilograms of material and "would not be practical to design $[5,6]$. Even lower enrichment is hypothetically possible, but as the enrichment percentage decreases the critical mass for unmoderated fast neutrons rapidly increases, with for example, an infinite mass of 5.4\% $235 \mathrm{U}$ being required [5]. For criticality experiments, enrichment of uranium to over $97 \%$ has been accomplished [7].

\section{ENRICHMENT METHODS}

Isotope separation is difficult because two isotopes of the same elements have very nearly identical chemical properties, and can only be separated gradually using small mass differences ( $235 \mathrm{U}$ is only $1.26 \%$ lighter than $238 \mathrm{U}$ ). This problem is compounded by the fact that uranium is rarely separated in its atomic form, but instead as a compound (235UF6 is only $0.852 \%$ lighter than 
238UF6.) A cascade of identical stages produces successively higher concentrations of 235U. Each stage passes a slightly more concentrated product to the next stage and returns a slightly less concentrated residue to the previous stage. There are currently two generic commercial methods employed internationally for enrichment: gaseous diffusion (referred to as first generation) and gas centrifuge (second generation) which consumes only $2 \%$ to $2.5 \%$. As much energy as gaseous diffusion, with centrifuges being at least a "factor of 20 " more efficient [8]. The throughput per centrifuge unit is very small compared to that of a diffusion unit so small, in fact, that it is not compensated by the higher enrichment per unit. To produce the same amount of reactor-grade fuel requires a considerably larger number (approximately 50,000 to 500,000 of centrifuge units than diffusion units. This disadvantage, however, is outweighed by the considerably lower (by a factor of 20) energy consumption per SWU for the gas centrifuge [9]. Later generation methods will become established because they will be more efficient in terms of the energy input for the same degree of enrichment and the next method of enrichment to be commercialized will be referred to as third generation. Some work is being done that would use nuclear resonance; however there is no reliable evidence that any nuclear resonance processes have been scaled up to production. Many studies had been done for separation laser advances $[10,11,12]$. Some isotopically purified elements are used in smaller quantities for specialist applications, especially in the semiconductor industry, where purified silicon is used to improve crystal structure and thermal conductivity. And carbon with greater isotopic purity to make diamonds with greater thermal conductivity. Isotope separation is an important process for both peaceful and military nuclear technology, and therefore the capability that a nation has for isotope separation is of extreme interest to the intelligence community [13]. A large number of parameters contribute to the definition of a laser isotope separation (LIS) plant. These parameters are dimensions and operating conditions. In the optimization algorithm, the calculation of the objective function may be repeated thousands of times; therefore, while the (LIS) physics is sophisticated simple methods must be used to describe each elementary physical process. Uranium isotopes provided as an example; given the power of the tunable laser beams find the parameters which maximize the superlative work of a Uranium facility.

\section{PHYSICAL MODELS}

\subsection{Evaporation rate}

For a given set of evaporation device i.e. electron gun power, focusing. Crucible dimensions etc.... We use here an empirical relation between the mass flow rate $\mathrm{Q}$ and the electron beam power P of a gun:

$$
\mathrm{Q}=\mathrm{ap}+\mathrm{b}
$$

When several guns are arranged end to end in a pod, the individual mass flow rates are added and the result is smeared uniformly on the total length $L$ of the pod.

\subsection{Vapor expansion}

During the expansion, exchanges between internal and kinetic energy of atoms are likely to occur. Let the density of atoms in the fundamental state is $\mathrm{n}_{\mathrm{o}}$, and on metastable excited electronic states $\left(\mathrm{n}_{10}, \mathrm{n}_{\mathbf{2 0}}, \ldots\right)$. In addition, the velocity $\bar{V}$, temperatures $\left(\mathrm{T}_{\mathrm{r}}, \mathrm{T}_{\Theta}, \mathrm{T}_{\mathrm{z}}\right)$ and the degree of ionization are characterize the vapor. The problem for $n_{o}$ has been solved by Rosengard (1989) [14] with the help of a Monte Carlo code based on the particle test method.

The vapor is supposed to be emitted through a slit from an imaginary vapor chamber in equilibrium at temperature $\mathrm{T}_{\mathbf{v}}$ and density $\mathrm{n}_{\mathbf{v}}$. See Fig. 1 , if $\bar{n}, \bar{T}$, and $\bar{V}$ are mean values along a line MM', parallel to the slit and bounded by the same vertical planes, then the dimensional $\bar{n} / n_{v}, \bar{T} / T_{v}$, and $\bar{V} / V_{v}$ are a function of the source Knudsen number $\left(\mathrm{K}_{\mathbf{n}}\right)[14]$ : 


$$
K_{n}=\frac{1}{\sqrt{2} n_{v} \Pi d^{2} L_{v}}
$$

and the reduced coordinates $\mathrm{R} / \mathrm{L}$ and $\sigma$

where

$\mathrm{d}=$ the hard sphere diameter

$\mathrm{L}_{\mathrm{v}}=$ the width of the slit

During the expansion, the vapor temperature decreases and it can be expected that the metastable ratio $\left(\mathrm{n}_{10} / \mathrm{n}_{0}\right)$ also decreases, because the atoms are in local thermodynamic equilibrium in the collision are near the source and that the populations are frost out side this area.

If three color beams are used, then:

Metastable ratio $P_{a}=\frac{n_{0}}{n_{0}+n_{10}}$

If four colors beams are used, then:

Metastable ratio $P_{a}=\frac{n_{0}+n_{10}}{n_{0}+n_{10}+n_{20}}$

We consider here, the expansion of one sort of atoms only $\left(\mathrm{n}_{0}\right)$. From review concentrates on the laser ionization process, experimentally $T_{i}$ is found to be a few percent, and in this work it may be useful to eliminate these parasitic ions. In the example, we illustrate, we shall take $\mathrm{T}_{\mathrm{i}}=0$.

\subsection{Illumination and photo-ionization}

Fig. 2 illustrates the interaction zone as the dashed square area, where photons and atoms intersect. Consider only laser beams propagating in the vacuum.

In the origin plane let us inscribe the beam in a square frame of side (a) ( $a$ is the side of the square laser beams) which is the best fit of the contour of the beam.

At distance $\mathrm{z}$ we shall only consider the light remaining in the frame and neglect that part which diffracted outside the frame.

We are now able to build up the global light intensity map of the interaction zone by putting side by side the frames which correspond to the successive folding of the beams.

Assuming that the light intensity and phases maps at the origin plane are identical for the three (or four) colors laser beams and that they diffract in the same manner i.e. same wavelength.

Let $\mathrm{P}_{\mathrm{e}}$ and $\mathrm{P}_{\mathrm{i}}$ be the illumination and the photo-ionization ratios defined as follows:

$\mathrm{P}_{\mathrm{e}}$ : the probability for one atom entering the interaction zone (IZ) to be illuminated, or to impact a rich plate.

$\mathrm{P}_{\mathrm{i}}$ : the probability for an illuminated atom to be photo-ionized.

Then

$$
P_{e}=\int_{-d / 2}^{+d / 2} \frac{d x}{2} \int_{-\infty}^{+\infty} \int_{-\infty}^{+\infty} f\left(V_{r}, V_{\Theta}\right) P\left(V_{r}\right) P\left(V_{\Theta}\right) d V_{r} d V_{\Theta}
$$

Where

$$
f\left(V_{r}, V_{\Theta}\right)=\max \left[\frac{L_{I Z}}{\sqrt{V_{r}^{2}+V_{\Theta}^{2}}}, L\right]
$$




$$
P(V)=\sqrt{\frac{m}{2 \pi K T}} \exp \left[-\frac{m}{2 K T}(V-\bar{V})^{2}\right]
$$

$\mathrm{L}_{\mathrm{IZ}}$ : the chord length (i.e. the length that part of the trajectory which is illuminated).

$\mathrm{C}$ : the laser repetition rate.

D: the aperture of the IZ.

$\mathrm{K}$ : the Boltzmann constant.

$\mathrm{m}$ : the atomic mass.

$\mathrm{V}_{\mathrm{r}}, \mathrm{V}_{\Theta}$ : the velocity in spherical coordinate.

This expression dose not take into account the velocity $\mathrm{V}_{\mathrm{z}}$ in the axial direction and is therefore conservative.

$$
P_{i}=F(\Phi)
$$

A relation $P_{i}=F(\Phi)$ between the local $\mathrm{P}_{\mathrm{i}}$ and the total energy flux per laser pulse $\Phi\left(\mathrm{mj} / \mathrm{cm}^{2} /\right.$ pulse $)$ can be established by using a code solving the coherent Bloch Eq.'s [15]. A typical non dimensional curve is shown in Fig. 3 where $\Phi_{0}$ is the value for which $\mathrm{P}_{\mathrm{i}}=0.99$.

Then $P_{i}$ can be calculated by integration in each frame of side (a) at distance $(Z)$ from the origin plane and for a given repetition rate:

$$
P_{i}=G(Z, a, C)
$$

The total power of the tunable light $\mathrm{P}_{\mathrm{o}}=$ being constant, we have:

$$
P_{o}=a^{2} E \mathrm{C}
$$

Where $\mathrm{E}$ is the total energy per pulse given by:

$$
E=\int_{0}^{a} \Phi(x, y) d x d y
$$

Then final $\mathrm{P}_{\mathrm{i}}$ is the mean value of the $\mathrm{P}_{\mathrm{i}}$ of the individual frames of the (IZ).

Therefore, Eq. 10 is obtained by using propagation and coherent photon /atom interaction.

\subsection{Extraction charge exchange}

Consider an electrostatic ions extraction only, $\mathrm{P}_{\mathrm{E}}$ and $\mathrm{P}_{\mathrm{x}}$ are defined as follows: $\mathrm{P}_{\mathrm{E}}=$ the probability for a photon-ion to impact the rich plates regardless of the charge exchange [16]:

$$
P_{E}=\frac{\int_{0}^{t_{\text {max }}} I(t) d t}{N_{o} q}
$$

Where:

$I(t)$ : the rich current.

$\mathrm{N}_{\mathrm{o}}$ : the number of photo-ions created.

q: the electron charge.

The electronic temperature $T_{e}$ is an important data. The electrons gain energy by a thermalization process with the ions and by the excess energy of the photo-ionization. Typical values of $T_{e}$ are $0.1-0.2 \mathrm{eV}$ for Uranium [17].

The charge exchange is supposed to occur only in the dense plasma. The ${ }^{235} \mathrm{U}$ ions which exchange their charge in the sheath are supposed to be collected [18].

Hence:

$\mathrm{P}_{\mathrm{x}}$ : is the probability of no charge exchange [16]. 


$$
P=\frac{\int_{0}^{t_{\max }} e^{-\sigma_{x} n_{8} v t} I(t) d t}{\int_{0}^{t_{\max }} I(t) d t}
$$

Where:

$\sigma_{\mathrm{x}}$ : the charge exchange cross section.

$\mathrm{n}_{8}$ : the ${ }^{238} \mathrm{U}$ density.

$V$ : the thermal velocity.

$$
\begin{aligned}
& V=\sqrt{\frac{8 K Z \bar{T}}{\pi m}} \\
& \text { Where } \\
& \overline{\mathrm{T}}=\frac{1}{3}\left(\mathrm{Tr}+\mathrm{T}_{\Theta}+\mathrm{T}_{\mathrm{z}}\right)
\end{aligned}
$$

Therefore, the charge exchange is equivalent to a lack of selectivity. Then it could be written as:

$$
\begin{aligned}
& \mathrm{P}_{\mathrm{i}}^{5}=\mathrm{P}_{\mathrm{i}} \times \mathrm{P}_{\mathrm{x}} \\
& \mathrm{P}_{\mathrm{i}}^{8}=\left(1-\mathrm{P}_{\mathrm{x}}\right) \frac{n_{5} \mathrm{P}_{\mathrm{i}}}{\mathrm{n}_{8}}
\end{aligned}
$$

Where $\mathrm{P}_{\mathrm{i}}^{5}$ and $\mathrm{P}_{\mathrm{i}}^{8}$ are the equivalent photo-ionization ratios of ${ }^{235} \mathrm{U}$ and ${ }^{238} \mathrm{U}$.

\subsection{Sputtering}

The sputtering yield can be approximated by the relation [17]:

$$
\mathrm{y}=\alpha\left(\frac{V}{1000}\right) \beta \quad \mathrm{V}>\mathrm{V}_{\mathrm{o}}
$$

The constants $\alpha, \beta$ and the potential $\mathrm{V}_{\mathrm{o}}$ can be inferred from the work of Robinson [19] for self sputtering $(\alpha=2.2, \beta=0.7, \mathrm{Vo}=200 \mathrm{~V})$.

\subsection{Dilution ratios}

The dilution ratio $T_{d}$ is the probability for an atom entering the (IZ) and that will not undergo photo-ionization to be collected by the rich plates. The approximate expression for $T_{d}$ which neglects the possible collisions in the (IZ) is [17]:

$$
T_{d}=2 \int_{-d / 2}^{+d / 2} d x \int_{V_{\Theta} \min }^{+\infty} P\left(V_{\Theta}\right) d V_{\Theta}
$$

With

$\frac{V_{r}(x)}{V_{\Theta \min }}=\frac{H}{D_{h}-x}$

Where $\mathrm{P}\left(\mathrm{V}_{\Theta}\right)$ is taken from Eq. 7, the integration is per formed on the width of the diaphragm (d) and on the velocities directed towards the rich plates. 


\section{EXAMPLE}

Many studied have been done on the separation technique of different isotopes $[20,21,22,23,24]$.The block diagram of enrichment state is shown in Fig. 4a and the flow diagram of mass flow rates of isotopes for each branch is described in Fig. $4 \mathrm{~b}$. This example concerns the design of a hypothetical Uranium facility. It is simple for understanding of the objective function to the various parameters.

\section{Input data (Ref.15)}

Length

Mean density at R- $0.55 \mathrm{~m} \quad 10^{13}$ atom $/ \mathrm{cm}^{3}$

Number of interaction zones 1

\section{Parameters}

Geometrical parameters: (see Fig.2) $D_{b}, D_{h}, d, R, H$.

Operating conditions: $\mathrm{V}$ negative potential of the rich plates.

$\mathrm{C}$ : repetition rate of lasers.

a: side of the square laser beams.

\section{Constraints}

Production assay 3\% (Ref.15)

$\mathrm{D}_{\mathrm{h}}=\mathrm{D}_{\mathrm{b}}+(\mathrm{R}+\mathrm{H}) / \mathrm{R} \quad$ (radial plates)

$\mathrm{V} \leq \mathrm{V}_{\mathrm{o}}$

Maximum number of bean folding $\mathrm{N}_{\max }$

The last two are arbitrary constraints due for example to technical limitations.

\section{Objective function}

Superlative work (sw/year pull power)

There are eight parameters and two relations. Hence six parameters are free. The optimization is then achieved in four steps:

\section{a. Sensitivity to the potential $\mathrm{V}$}

Increasing the potential improves the extraction, but increases the sputtering and hence increases the tails assay. In almost all cases starting form mean configuration with constraints explained before with the selected value of $\mathrm{V}$.

\section{b. Sensitivity to the geometrical parameters}

Taking into account the constraints mentioned above and a set of $\mathrm{V}, \mathrm{C}, \mathrm{P}_{\mathrm{i}}, \mathrm{P}_{\mathrm{e}}$ values, three independent parameters are left, for instance $D_{b}, d$, and R.H. could be calculated in order to satisfy $D_{h}=D_{b}+(R+H) / R$ for each $d$, we can represent the objective as a function of $D_{b}$ and $R$..

For their optimal choice resulting from the combined effects of ions extraction, charge exchange and dilution rate.

\section{c. Management of the photons}

For given $d$ and $C$, the best way fill the interaction zone is to maximize the product $\mathrm{P}_{\mathrm{i}} \mathrm{P}_{\mathrm{e}}$. The number of folding is chosen in order to cover a rectangle of dimensions Lxh with:

$$
L \geq d \text { and } h \leq \frac{V_{r}+\sigma\left(V_{r}\right)}{C}
$$

Where $\sigma\left(\mathrm{V}_{\mathrm{r}}\right)$ is the mean square deviation of the radial velocity $\mathrm{Vr}$.

Increasing (a) improves $\mathrm{P}_{\mathrm{e}}$ (due to a limitation number of folding $\mathrm{N}$ ) and conversely degrades $\mathrm{P}_{\mathrm{i}}$ because the energy flux per pulse decreases. 
If we increase $\mathrm{C}$, we can decrease the height $\mathrm{h}$ of the (IZ) and correlatively the side of the frame (a). On the other hand decreasing (a) involved more diffraction.

Hence while for increasing $C, P_{E}$, and $P_{x}$ are improved, $P_{i} P_{e}$ decreases due to the diffraction. An optimum of $\mathrm{C}$ is then expected.

\section{d. Global optimization}

Several trials, starting from different initial sets of parameters, were necessary to find the optimum.

\section{CONCLUSION}

This work uses simple methods to describe the elementary physical processes. Uranium is given as an example. The optimization is spilt in four steps for a better understanding of the sensitivity of the objective to different groups of parameters. This conclusion depends on the input data of this particular problem and obviously it must not be extended to the design of the plant.

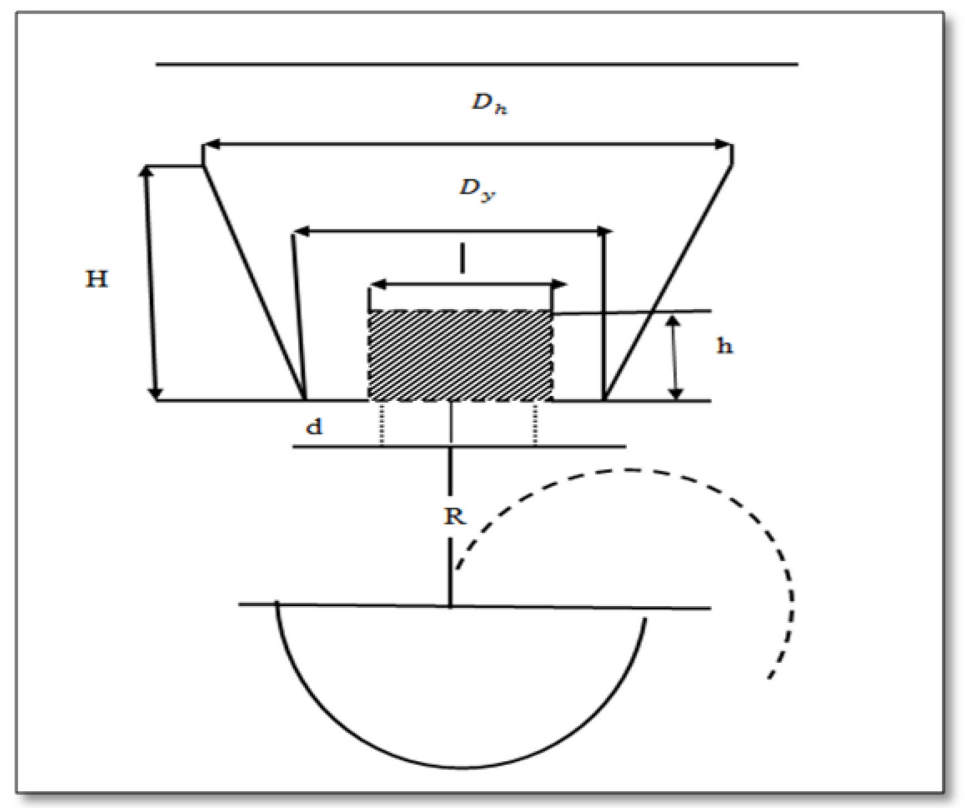

Fig. 1. Axial geometry for vapor expansion calculation.

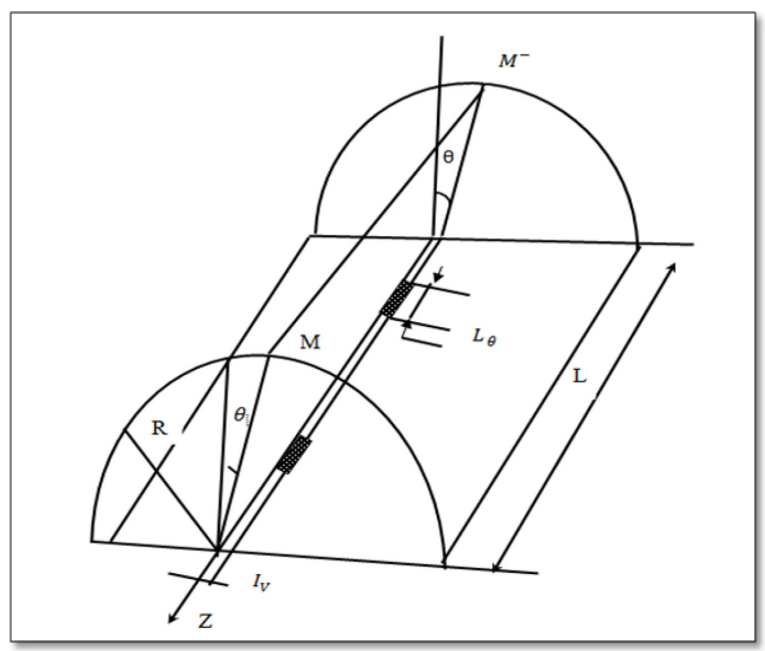

Fig. 2. Cross section of a separator showing the main dimension. 


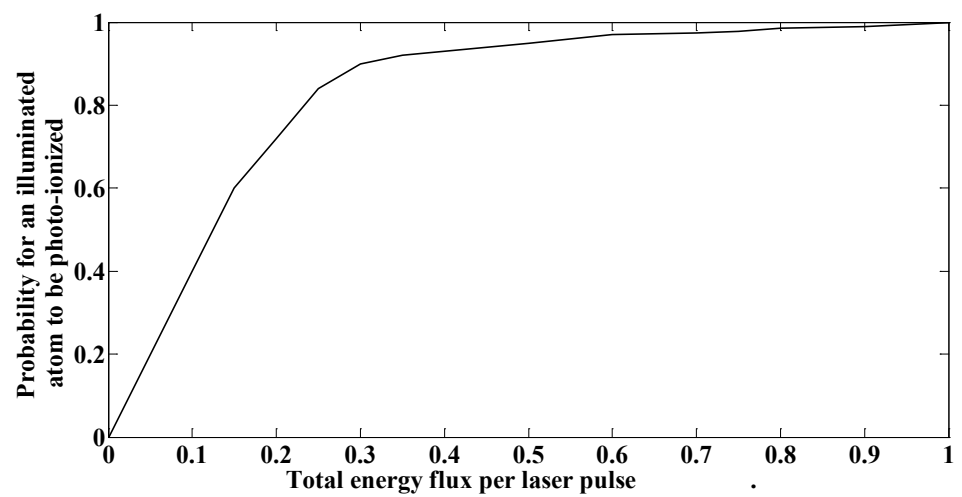

Fig. 3. Photoionization rate $\rho$ i as a function of total tunable light power Ref. 15.

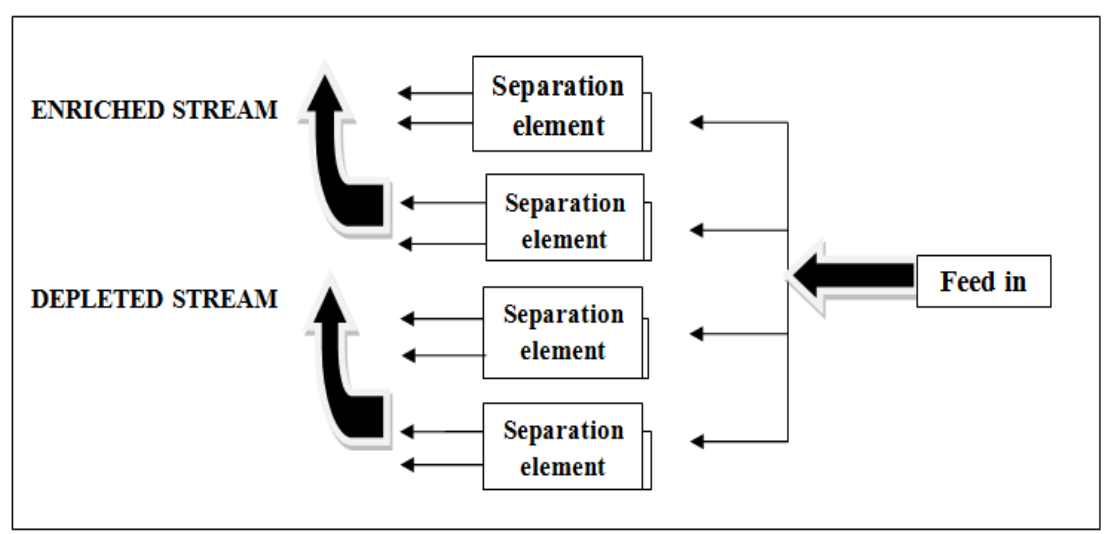

Fig. 4a. Block diagram of enrichment stage

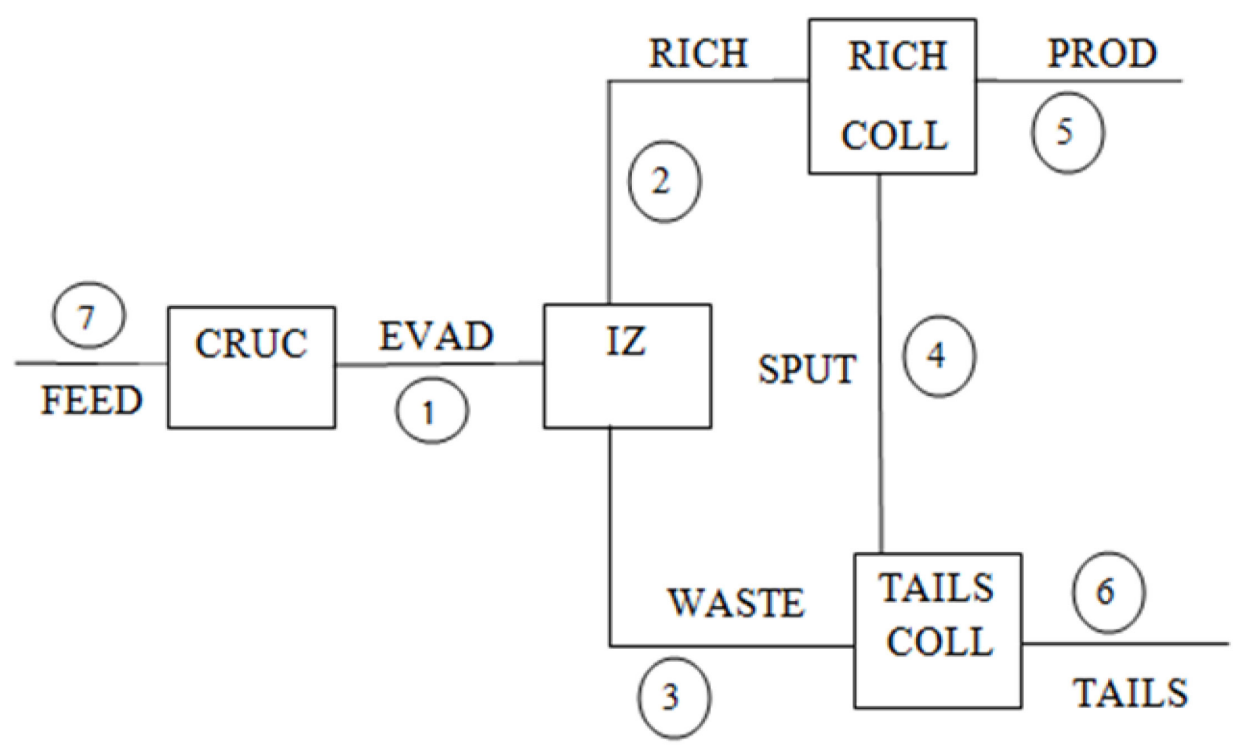

Fig. 4b. Flow sheet diagram (4 capacities -7 branches) 


\section{References}

[1] S. Richter, A. Alonso, W.De bolle, R.Wellum, and P.D.P. Taylor, “Isotopic Finger prints' for Natural Uranium Ore Samples, “International Journal of Mass Spectrometry. 193 (1999) 9-14.

[2] U.S. Department of Energy Nuclear Material Safeguards for Uranium Enrichment Plats. Technical Publication No. ISPO-347/R6. (Oak Ridge, TN, Oak Ridge National Laboratory. (2005).

[3] OECD Nuclear Energy Agency (2003). Nuclear Energy Today. OECD Publishing. ISBN 9789264103283 (2003) 25.

[4] Alexander Glaser (6 November 2005). "About the Enrichment Limit for Research ReactorConversion : Why 20\%?" (PDF). Princeton University. Retrieved 18 April (2014).

[5] Forsberg, C. W.; Hopper, C. M.; Richter, J. L.; Vantine, H. C. (March 1998). "Definition of Weapons-Usable Uranium-233" (PDF). ORNL/TM-13517. Oak Ridge National Laboratories. Retrieved 30 October (2013).

[6] Sublette, Carey (4 October 1996). "Nuclear Weapons FAQ, Section 4.1.7.1: Nuclear Design Principles - Highly Enriched Uranium". Nuclear Weapons FAQ. Retrieved 2 October (2010).

[7] Mosteller, R.D. (1994). "Detailed Reanalysis of a Benchmark Critical Experiment: WaterReflected Enriched-Uranium Sphere" (PDF). Los Alamos technical paper (LA-UR-93-4097): 2. Retrieved 19 December (2007). The enrichment of the pin and of one of the hemispheres was 97.67 w/o, while the enrichment of the other hemisphere was $97.68 \mathrm{w} / \mathrm{o}$.

[8] http://www.world-nuclear.org/info/ Nuclear-Fuel-Cycle/ Conversion-Enrichment-andFabrication/ Uranium-Enrichment/\#.UWrver-IRAs

[9] GE Hitachi Nuclear Energy Selects Wilmington, N.C. as Site for Potential Commercial Uranium Enrichment Facility". Business Wire. 30 April 2008. Retrieved 30 September (2012).

[10] Broad and J. William (20 August 2011). "Laser Advances in Nuclear Fuel Stir Terror Fear". The New York Times. Retrieved 28 August (2012).

[11] Alexander Glaser, "About the Enrichment Limit for Research Reactor Conversion: Why $20 \%$ ?", The $27^{\text {th }}$ International Meeting on Reduced Enrichment for Research and Test Reactors (RERTR), November 6-10 (200), Bosten, Maassachusetts. Princeton University. Retrieved 18 April (2014).

[12] F. A. Lindemann, and F. W. Aston (1919). "The possibility of separating isotopes". Philosophical Magazine Series 6 (Taylor\& Francis Group) 37 (221) 523-534. Doi: 10.1080/14786440508635912. Retrieved 17 January (2014).

[13] Thomas and Andrew (November 30, 2000). "AMD tests 'super silicon' to beat heat problems". The Register: Channel. The Register. Retrieved 17 January (2014).

[14] A. Rosengard, Progress in Astronautics and Aeronautics, 116 (1989).

[15] M. Sargent and M.O. Scully, laser Physics, Addison Wesley ed. (1989).

[16] F.F. Chen, Phys. Fluids, 25(12) December (1989).

[17] F. Doneddu, Second workshop-separation phenomena in liquids and gases-Versailles, July 1012 (1989).

[18] J. F. Lizee and M. Alexander, Internal Report, Reported in International Symposium on Isotope Separation and Chemical Exchange Uranium Enrichment Tokyo (JP) 28 Oct - 1 Nov (1990).

[19] M. T. J. Robinson, Appl. Phys. 54 (5) (1983). 
[20] J. R. Lamarsh and A. J. Baratta A, Introduction to Nuclear Engineering, Third edition, Prentice-Hall Inc. (2001).

[21] H. G. Wood and A, Glaser, The Gas Centrifuge and Nuclear Weapons Proliferation, Physics Today (2008).

[22] A. G. Belikov and V. G. Papkovich, Problems of Atomic Science and Technology, 52 (2009) 57.

[23] J.R. Beckerman, Beginning the Search for the Inverse Fission of Uranium, M.Sc. Thesis, Oregon State University, June (2010).

[24] F. Caron and S. Smith, Chem. listy, 104 (2010) 222. 